ACCEPTED MANUSCRIPT

\title{
A motion correction approach for oral and maxillofacial cone-beam CT imaging
}

To cite this article before publication: Tao Sun et al 2021 Phys. Med. Biol. in press https://doi.org/10.1088/1361-6560/abfa38

\section{Manuscript version: Accepted Manuscript}

Accepted Manuscript is "the version of the article accepted for publication including all changes made as a result of the peer review process, and which may also include the addition to the article by IOP Publishing of a header, an article ID, a cover sheet and/or an 'Accepted

Manuscript' watermark, but excluding any other editing, typesetting or other changes made by IOP Publishing and/or its licensors"

This Accepted Manuscript is (c) 2021 Institute of Physics and Engineering in Medicine.

During the embargo period (the 12 month period from the publication of the Version of Record of this article), the Accepted Manuscript is fully protected by copyright and cannot be reused or reposted elsewhere.

As the Version of Record of this article is going to be / has been published on a subscription basis, this Accepted Manuscript is available for reuse under a CC BY-NC-ND 3.0 licence after the 12 month embargo period.

After the embargo period, everyone is permitted to use copy and redistribute this article for non-commercial purposes only, provided that they adhere to all the terms of the licence https://creativecommons.org/licences/by-nc-nd/3.0

Although reasonable endeavours have been taken to obtain all necessary permissions from third parties to include their copyrighted content within this article, their full citation and copyright line may not be present in this Accepted Manuscript version. Before using any content from this article, please refer to the Version of Record on IOPscience once published for full citation and copyright details, as permissions will likely be required. All third party content is fully copyright protected, unless specifically stated otherwise in the figure caption in the Version of Record.

View the article online for updates and enhancements. 


\section{A Motion Correction Approach for Oral and Maxillofacial Cone-beam CT Imaging}

Tao Sun ${ }^{1}$, Reinhilde Jacobs ${ }^{2}$, Ruben Pauwels ${ }^{3}$, Elisabeth Tijskens ${ }^{2}$, Roger Fulton ${ }^{4,5}$ and Johan Nuyts ${ }^{6}$

${ }^{1}$ Paul C. Lauterbur Research Center for Biomedical Imaging, Shenzhen Institute of Advanced Technology, Chinese Academy of Science, Shenzhen, China

${ }^{2}$ OMFS-IMPATH, Department of Imaging and Pathology, KU Leuven, Leuven, Belgium

${ }^{3}$ Aarhus Institute of Advanced Studies, Aarhus University, Denmark

${ }^{4}$ School of Health Sciences, University of Sydney, Sydney, Australia

${ }^{5}$ Department of Medical Physics, Westmead Hospital, Westmead, Australia

${ }^{6}$ Medical Imaging Research Center and Department of Nuclear Medicine, KU

Leuven, Leuven, Belgium

Abstract. Patient movement affects image quality in oral and maxillofacial conebeam CT imaging. While many efforts are made to minimize the possibility of motion during a scan, relatively little attention has been given to motion correction after the acquisition. We propose a novel method which can improve the image quality after an oral and maxillofacial scan. The proposed method is based on our previous work and is a retrospective motion estimation and motion compensation (ME/MC) approach that iteratively estimates and compensates for rigid pose change over time. During motion estimation, image update and motion update are performed alternately in a multiresolution scheme to obtain the motion. We propose to use a feature-based motion update and patch-based image update in the iterative estimation process, to alleviate the effect of limited scan field-of-view on estimation. During motion compensation, a fine-rèsolution image reconstruction was performed with compensation for the estimated motion. The proposed ME/MC method was evaluated with simulations, phantom and patient studies. Two experts in dentomaxillofacial radiology assessed the diagnostic importance of the resulting motion artifact suppression. The quality of the reconstructed images was improved after motion compensation, and most of the image artifacts were eliminated. Quantitative analysis by comparison to a reference image and by calculation of a sharpness metric agreed with the qualitative observation. The results are promising, and further evaluation is required to assess the clinical value of the proposed method.

Keywords: oral and maxillofacial imaging, motion estimation, motion compensation, cone-beam Computed Tomography (CBCT) 


\section{Introduction}

Patient motion is one of the main causes of image artifacts in oral and maxillofacial imaging. Unlike clinical helical CT, dental imaging uses cone-beam CT (CBCT) most commonly, which typically requires longer scan time and thus has a higher probability of patient movement. It has been shown that, due to fear of the tube/detector movement, some patients, particular children, move their head during a scan $[1,2]$. Also, it is difficult to prevent aged patients with brain disorders such as Parkinson's disease from moving [3]. In a study, it was found that about $18 \%$ of the pediatric patients and $24 \%$ of aged subjects were likely to move sufficiently to induce motion artifacts in the reconstructed images [4].

The motion artifacts in the reconstructed image can degrade the image quality, adversely affecting diagnosis or treatment planning [5]. Even when the motion is too small to be noticed directly, it may still cause a significant resolution loss in the reconstructed image. In such cases, motion, rather than the resolution of the flat panel detector, may become the limiting factor of the image resolution. Further problems arise when metal implants are present together with motion [6]. To obtain an image without artifacts, a retake of the scan may be required, which inevitably causes additional radiation dose to the subject.

Many efforts have been made to prevent patient motion. It is often suggested an examiner should instruct the subjects to close their eyes and be prepared for the scanner movement prior to the acquisition. Patients are sometimes immobilized using a head strap, and/or a chin holder. However, solely relying on the hardware to fix the head may not prevent all potential movements [7]. There are several existing retrospective correction methods focusing on general CBCT applications. Wicklein et al [8], Sisniega et al [9], Jang et al. [10] and Bruder et al. [11] minimize an image-based cost function (which essentially detects motion artifacts) to estimate the motion of extremities, head and lung. Ouadah et al. [12] performed motion correction for head imaging by estimating and compensating for the rigid motion through a 2D-3D registration process, under the precondition that a prior 3D motion-free image of the same subject was available. Ko et al. [13] proposed a generalized CT motion correction framework using a deep neural network, which is capable of real-time correction. However, their model has limited ability to compensate for fast out-of-plane motion and was not applied to any clinical data.

On the other hand, relatively few works have been published on methods, specifically for reducing motion artifacts in reconstructed oral and maxillofacial CBCT images. One recent work demonstrated the potential to track the head movement with the help of an optical camera [14]. Another work aimed to estimate and compensate for the motion retrospectively, by enforcing the locally consistent contours of teeth [15]. We previously described a retrospective method to estimate and compensate for head motion in helical CT imaging, by maximizing the data-consistency between the object and projections [16]. Here, by accounting for the inherent differences between oral and 
(a)

maxillofacial CBCT and helical CT, we propose a novel method for oral and maxillofacial imaging. The proposed method was evaluated using simulations, a phantom scan, and a patient scan with motion artifacts. Both quantitative and qualitative analysis were performed. Additionally, two experienced dentists trained in dentomaxillofacial radiology further validated the clinical applicability of the proposed method.

\section{Materials and Methods}

(b)

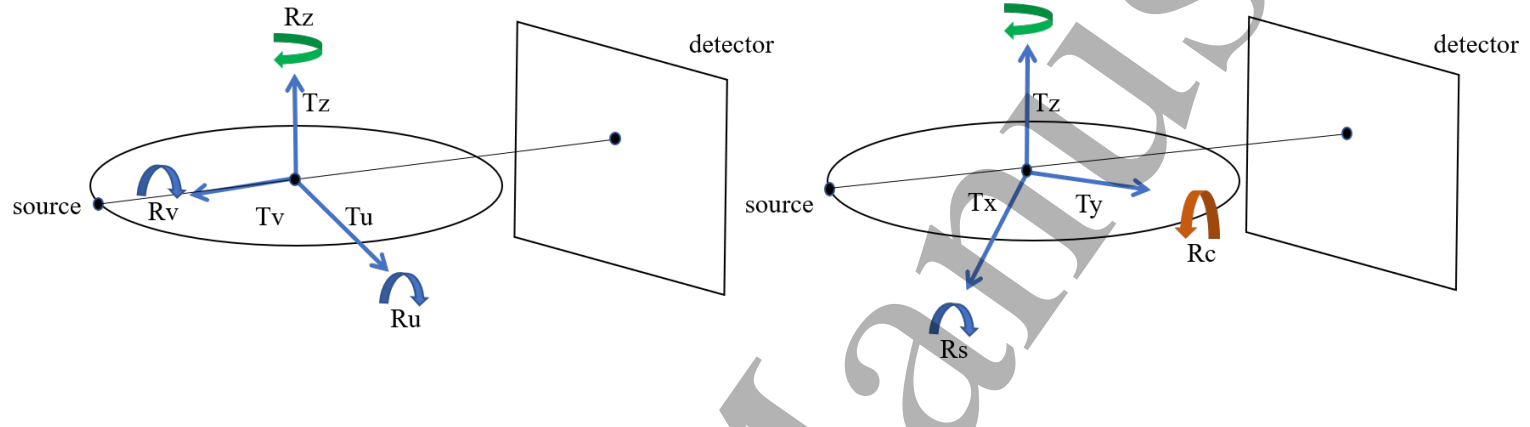

Figure 1. A dental CBCT system usually has a flat detector surface. For each view, the origins of (a) the detector and (b) scanner coordinate systems coincide. $\mathrm{R}$ denotes rotation, $\mathrm{T}$ denotes translation.

Let us first define the coordinate systems of a virtual CBCT imaging system in Figure 1. We define two coordinate systems. One is the detector coordinate system (Figure 1a), which is fixed with respect to the rotating source-detector pair, and its z-axis coincides with scanner rotation axis. Another is the world coordinate system (Figure 1b), which is fixed with respect to the scanner. For one projection view, we may define a rigid transformation in the scanner system as:

$$
\mathrm{S}_{\mathrm{world}}=\left[\mathrm{R}_{\mathrm{t}}, \mathrm{R}_{\mathrm{s}}, \mathrm{R}_{\mathrm{c}}, \mathrm{T}_{\mathrm{x}}, \mathrm{T}_{\mathrm{y}}, \mathrm{T}_{\mathrm{z}}\right]^{\mathrm{T}}
$$

which can be mapped to the detector system as:

$$
\mathrm{S}_{\text {detector }}=\left[\mathrm{R}_{\mathrm{u}}, \mathrm{R}_{\mathrm{v}}, \mathrm{R}_{\mathrm{z}}, \mathrm{T}_{\mathrm{u}}, \mathrm{T}_{\mathrm{v}}, \mathrm{T}_{\mathrm{z}}\right]^{\mathrm{T}}
$$

where $T_{u}$ and $T_{v}$ are translations tangent and orthogonal to the detector. The aim is first to estimate the pose of the head at each acquired CBCT projection, which we here refer to as motion estimation (ME); then compensate for the estimated motion during the final image reconstruction process, which we refer to as motion compensation (MC). Figure 2 shows the proposed motion estimation and motion compensation (ME/MC) algorithm schematically. $\mathrm{ME}$ is an iterative process, in which one iteration contains one motion update and one image update. A similar coordinate system definition and correction scheme to that in Figure 2 was applied to helical scan in [16].

Oral and maxillofacial CBCT typically involves a small field of view and the acquired projections suffer from a strong transaxial truncation, whereas transaxial truncation is usually minor or absent in helical CT. Since ME/MC involves an iterative 
process with re-projection, insufficient information outside the completely sampled region will induce errors. There is value in minimizing the amount of computation devoted to the background region, which can be done without compromising the accuracy of the motion estimates. More details of the ME and MC implementations are given below.

\subsection{Motion update in $M E$}

An initial image is first reconstructed from the measured projections without any motion correction. As in our previous work, we assumed that the rigid pose of the measured object may be different for each projection view. Consequently, a rigid transformation representing the object pose change was estimated for every view, by a 2D-3D registration process. Let $r_{0}$ be the current estimate of the one of these parameters (a rotation or translation), $r$ is the value to be estimated for that parameter. Assuming that the actual change in the pose parameter represented by $\widehat{r}$ is small, the derivative of projection with respect to $r$ can be approximated as a finite difference of the intensities. For that purpose, we introduce auxiliary projections which are derived by reprojecting the current image estimate with a small change $\Delta r$ applied to one of the motion parameters. Auxiliary projections are computed to construct the least squares 
minimization problem at one view for given iteration:

$$
\widehat{r}=\underset{r}{\operatorname{argmin}}\left\|\Delta r\left(\mathrm{~F}_{\text {meas }}-\mathrm{F}_{\text {reproj }}\right)-r\left(\mathrm{~F}_{\text {incre }}-\mathrm{F}_{\text {reproj }}\right)\right\|^{2}
$$

where $\Delta r$ is induced increment in $r, \mathrm{~F}_{\text {meas }}$ is the measured projection, $\mathrm{F}_{\text {reproj }}$ is the re-projection of the current image estimate, and $\mathrm{F}_{\text {incre }}$ is the auxiliary projection of the image, computed with the small change $\Delta r$ applied to $r$. Eq. (3) enables an approximate analytical calculation of one degree-of-freedom motion parameter at each iteration:

$$
\begin{aligned}
& \widehat{r}=\frac{\sum_{\mathrm{N}}(\mathrm{P} \cdot \mathrm{Q})}{\left\|\sum_{\mathrm{N}} \mathrm{Q}^{2}\right\|} \Delta r \\
& \text { where } \mathrm{P}=\mathrm{F}_{\text {meas }}-\mathrm{F}_{\text {reproj }}, \quad \mathrm{Q}=\mathrm{F}_{\text {incre }}-\mathrm{F}_{\text {reproj }}
\end{aligned}
$$

where $\mathrm{N}$ is the total number of pixels in projection view $\theta$. We found the translation $T_{v}$ results in very small magnification of the projection, which can be ignored without adverse effects on final motion corrected image. The newly estimated parameter $\widehat{r}$ was added to the current estimate $r_{0}$ immediately when éstimating the value of the next parameter. The remaining five rigid parameters were estimated in a sequential way at each given projection view (first translation, then rotation). The influence of the induced parameter change on projection was constrained to one degree-of-freedom only each time. This motion estimate for all projection views completes the update of the rigid motion at one iteration, by adding to the current motion estimate. Above process was labeled as "motion update" in Figure 2. Simulations were performed to determine a value of $\Delta r$ for each degree-of-freedom to be estimated that provided an acceptable balance of speed and accuracy. Excessively large values of $\Delta r$ were observed to reduce the number of iterations required, thus improving speed, but compromise the accuracy of estimates. Excessively small values increased the number of iterations without improving accuracy. The exact value for each degree-of-freedom used was the largest that did not conpromise the estimation accuracy. In this study, $\Delta r$ was $0.5 \mathrm{~mm}$ for all translations and 0.5 degree for all rotations. Above procedure is similar to the one in [16], in which details about the derivation of Eq. (4) can be found.

Our previous method assumed no transaxial truncation in a scan. As explained earlier, this is no longer valid for an oral and maxillofacial scan, as the object outside the region-of-interest (ROI) does not contribute to all acquired views. The truncation artifacts typically result in blurring across the missing projection lines. By focusing on high frequency contents, we expect to reduce the influence of missing projection lines on the motion estimation. Therefore, in this study we did not derive the motion based on the projections themselves but on the high-frequency parts of them.

To do so, we introduced a feature-based motion update. We modified the original motion update by introducing a Laplacian of Gaussian (LOG) operation on all projections (measured projections, re-projections, auxiliary projections). For a given 2D projection view, we applied the LOG operator by applying the convolution kernel:

$$
\operatorname{kernel}(\mathrm{x}, \mathrm{y})=-\frac{1}{\pi \sigma^{4}}\left[1-\frac{x^{2}+y^{2}}{2 \sigma^{2}}\right] \exp \left(-\frac{x^{2}+y^{2}}{2 \sigma^{2}}\right)
$$


where $\sigma$ is the Gaussian smoothing width. A discrete Laplacian kernel that approximates above function was used in the following study, where the 2D window size can be defined. Therefore, the minimization problem in Eq. (3) becomes:

$$
\begin{aligned}
& \widehat{r}=\underset{r}{\operatorname{argmin}} \| \Delta r\left(\operatorname{LOG}\left\{\mathrm{P}_{\text {meas }}\right\}-\operatorname{LOG}\left\{\mathrm{P}_{\text {reproj }}\right\}\right) \\
& -r\left(\operatorname{LOG}\left\{\mathrm{P}_{\text {incre }}\right\}-\operatorname{LOG}\left\{\mathrm{P}_{\text {reproj }}\right\}\right) \|^{2}
\end{aligned}
$$

where LOG denotes the Laplacian of Gaussian operation described above. The rest of the estimation process remained unchanged. In addition, first order gradients, i.e. Sobel and Roberts operators, were applied instead in Eq. (6) to be compared with the LOG filter. The one with the optimal setup that resulted in the best recovered image quality was selected in this study.

\subsection{Image update in $M E$}

After obtaining the motion, the image can be updated within an iterative reconstruction process. First let us define the forward model of the acquisition. Assuming monochromatic radiation and ignoring scattering effects, the penalized Poisson loglikelihood function for the attenuation image $\mu$ is:

$$
\mathrm{L}(\mu)=\sum_{i} y_{i} \ln \bar{y}_{i}(\mu)-\bar{y}_{i}(\mu)-\beta \mathrm{X}(\mu)
$$

where $i$ is the index of the projection lines, $y_{i}$ is the measured transmission scan at $i$, $\bar{y}_{i}(\mu)$ is the estimated transmission scan at $i$ computed from the attenuation image $\mu$, $\mathrm{X}(\mu)$ is the regularization term and $\beta$ is the weighting factor. We set $\beta=0$ in this study to ignore the usage of any prior.

By maximizing Eq. (7), one can find the optimal attenuation image iteratively. For example, ignoring the regularization term, the update equation of Maximum Likelihood Transmission-Reconstruction (MLTR) [17] is:

$$
\mu_{j}^{\text {new }}=\mu_{j}+\frac{\sum_{i} c_{i j}\left(\bar{y}_{i}-y_{i}\right)}{\sum_{i} c_{i j}\left(\sum_{k} c_{i k}\right) \bar{y}_{i}}
$$

where $\mu_{j}$ is the linear attenuation coefficient at voxel $j, c_{i j}$ is the intersection length of projection line $i$ with voxel $j$. Note that we drop the argument of $\bar{y}_{i}$ for clarity. Compensating for the motion can be done by adjusting the system matrix on-the-fly. Instead of moving the reconstructing image in every view, motion compensation was done by considering a coordinate system fixed to the object and incorporating the motion (now associated to the source-detector pair) into the system matrix. This corresponds to an arbitrary 3D motion of a virtual gantry around the object being scanned, created by the superposition of the inverse of the object motion on the X-ray source trajectory.

To minimize the amount of computation devoted to the background region without compromising the accuracy of motion estimates, we propose to use patchbased reconstruction in image updates. Patch-based reconstruction approaches have been proposed for various applications in CT imaging [18-20]. The idea is that a reconstruction volume can be divided into important patches and not-so-important 

allows us to focus the computations more on the interesting regions. We defined two patches in a scan (Figure 3): high-res patch - the patch containing the fully sampled ROI where a smaller voxel size was used; and a low-res patch - the patch comprising the remainder of the object where a coarser resolution model (e.g., voxel size was 4 times larger in all 3 dimensions) was used. The modified update equation of MLTR with the capability to account for patches is:

$$
\mu_{j}^{\text {new }}=\mu_{j}+\frac{a_{j} \sum_{i} c_{i j}\left(\bar{y}_{i}-y_{i}\right)}{\sum_{i} c_{i j}\left(\sum_{k} c_{i k} a_{k}\right) \bar{y}_{i}}
$$

where

$$
\left\{\begin{array}{l}
a_{j}=1 \text { if } j \in \text { patch } \\
a_{j}=0 \text { if } j \notin \text { patch }
\end{array}\right.
$$

The patches were updated sequentially, each patch being considered as a group of voxels in a grouped coordinate algorithm. The voxel weighting factor $a_{j}$ also plays an important role in the convergence of the attenuation values at each voxel. Sequentially updating groups of voxels is known to improve convergence, as the denominator of the update steps will be smaller when the area of the updated patch is smaller [18]. For the one subiteration update in MLTR, we have two consecutive steps (Figure 3): a first update performed only on the high-res patch, and a sequential update performed on low-res patch. MLTR performed such alternate updates for two patches to complete the image update in ME. For motion update, the re-projection process involved the forward projections with different resolutions for different patches, and combination of these projections into one projection.

\subsection{Multi-resolution acceleration in $M E$}

The motion update and image update were alternated to increase the likelihood, and the iterations were stopped when the summation of projection errors did not change by more than $2 \%$. These errors between the reprojected and measured data were calculated over all projection views. A multi-resolution approach was applied to accelerate the computation. For example, the starting image resolution level was $4 \times 4 \times 4$, i.e. a downsampling factor of four was applied in all directions. There was a resampling with a factor of two between adjacent levels. So the image resolution at next level would be $2 \times 2 \times 2$. A similar multi-resolution approach was applied in the projection domain. Since the motion estimate hardly changed during the computations at the finest resolution, we stopped the scheme at the second finest resolution, which significantly reduced the computation. The parameter settings and exact iteration numbers at each resolution level of the multi-resolution scheme for the studies below are provided in Figure 4 and Table 1 . 


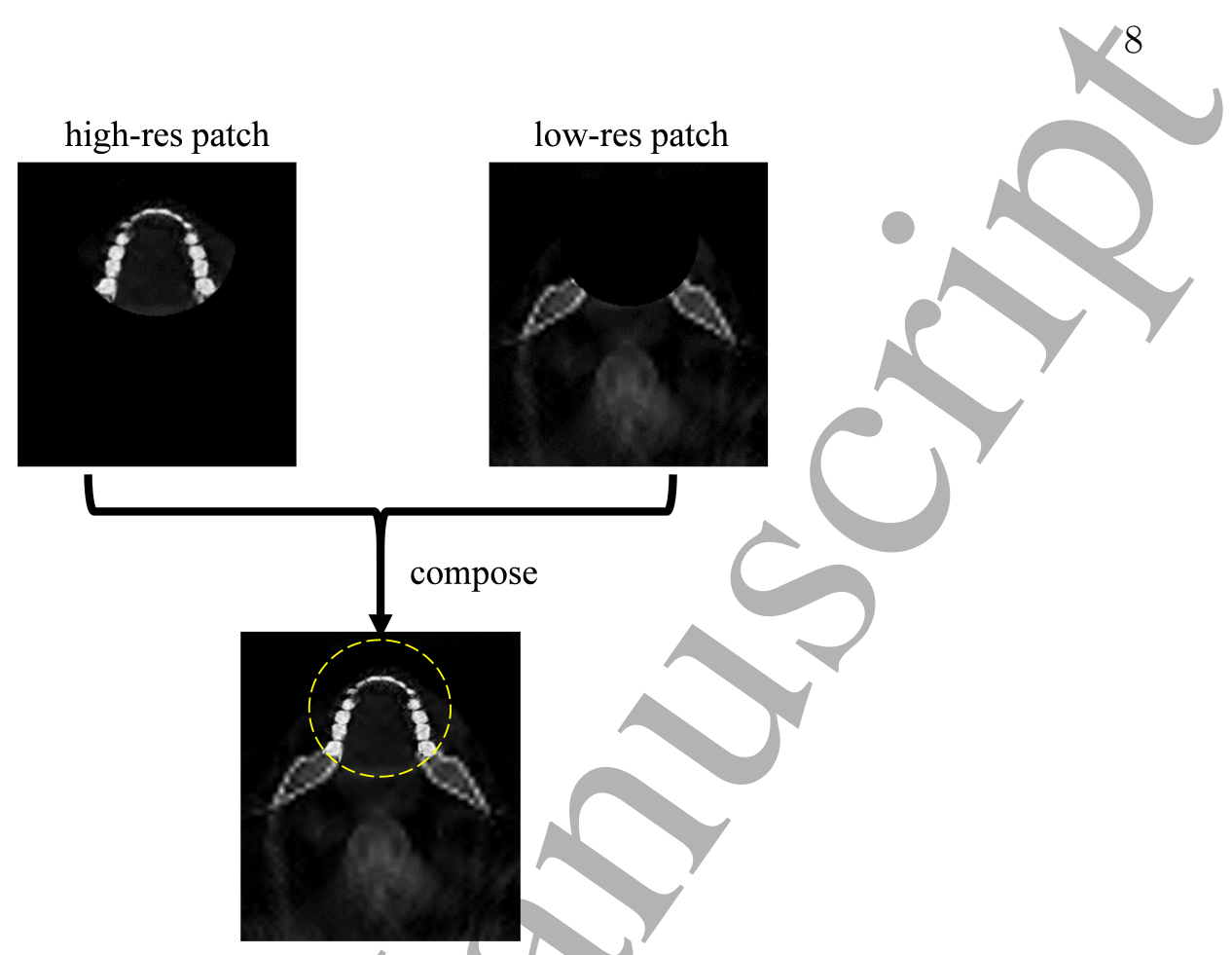

Figure 3. Schematic representation of an iteration of the image update. A first reconstruction update was only performed in the high-res patch, and a sequential reconstruction update was performed in the low-res patch. Then these two patches were combined into a single image. The dashed circle indicates the fully sampled field-of-view (FOV), i.e. high-res patch.

Multi-resolution image update

$\begin{array}{ll}\text { Level } 3120 \times 120 \times 120 & 1 \text { OSEM-iteration } 30 \text { subsets } \\ \text { Level } 2240 \times 240 \times 240 & 1 \text { OSEM-iteration } 40 \text { subsets } \\ \text { Level } 1481 \times 481 \times 481 & \text { No updates }\end{array}$

Figure 4. Example multi-resolution update implemented in this study. The number of OSEM-iterations and subsets were applied for the image update at each resolution level are listed. Note that we stop the estimation at the second last level, hence no image update were computed at level 1.

\subsection{Final reconstruction in $M C$}

On completion of the iterative ME process, a final reconstruction was performed using the estimated motion. For this reconstruction, a full iterative algorithm can be used that incorporates the motion. Alternatively, to speed up the calculation, an approximate circular Feldkamp-Davis-Kress (FDK) algorithm [21] was implemented, where a first order motion compensation is obtained by taking the motion for each view into account in the back-projection step. This approximation could be applied because the use of physieal restraints limited average motion amplitude, which is typically around $0 \sim 3$ $\mathrm{mm}[14]$. 


\subsection{Quantitative analysis}

For the simulation and phantom studies, we computed the root-mean-square error (RMSE), mean structural similarity index measure (MSSIM) [22] and gradient variance (GV) $[9,23]$ for both uncorrected and corrected images with respect to a reference image, which was reconstructed from a motion-free scan. Taking the motion-corrected image (MCI) as example, RMSE was calculated as:

$$
\operatorname{RMSE}(\mathrm{REF}, \mathrm{MCI})=\sqrt{\frac{1}{\mathrm{~N}} \sum_{\mathrm{j}}\left(\mu_{\mathrm{REF}, \mathrm{j}}-\mu_{\mathrm{MCI}, \mathrm{j}}\right)^{2}}
$$

where $\mu_{j}$ represented the intensity of voxel $\mathrm{j}$, and $\mathrm{N}$ is the total number of voxels. MSSIM index was calculated as:

$$
\begin{aligned}
& \operatorname{MSSIM}(\mathrm{REF}, \mathrm{MCI})=\frac{1}{\mathrm{~N}} \sum_{\mathrm{j}} \operatorname{SSIM}\left(\mu_{\mathrm{REF}, \mathrm{j}}, \mu_{\mathrm{MCI}, \mathrm{j}}\right) \\
& \text { where } \operatorname{SSIM}\left(\mu_{\mathrm{REF}}, \mu_{\mathrm{MCI}}\right)=\frac{\left(2 \bar{\mu}_{\mathrm{REF}} \bar{\mu}_{\mathrm{MC}}+\mathrm{c}_{1}\right)\left(2 \sigma_{\mathrm{REF}-\mathrm{MC}}+\mathrm{c}_{2}\right)}{\left(\bar{\mu}_{\mathrm{REF}}^{2}+\bar{\mu}_{\mathrm{MC}}^{2}+\mathrm{c}_{1}\right)\left(\sigma_{\mathrm{REF}}^{2}+\sigma_{\mathrm{MC}}^{2}+\mathrm{c}_{2}\right)}
\end{aligned}
$$

$\bar{\mu}$ and $\sigma$ represent the mean intensity and the variance of the patch, $\sigma_{\mathrm{REF}-\mathrm{MC}}$ is the covariance of two patches. The constants $c_{1}=10^{-4} / \mathrm{cm}^{-2}$ and $c_{2}=3 \times 10^{-4} \mathrm{~cm}^{-2}$ were used to stabilize the low intensities. GV, which was shown to be sensitive to motion artifacts, was computed as:

$$
\begin{aligned}
& \operatorname{GV}(\mu)=\sum_{j}\left(\operatorname{TV}\left(\mu_{j}\right)-\frac{1}{N} \sum_{j} \operatorname{TV}\left(\mu_{j}\right)\right)^{2} \\
& \text { where } \operatorname{TV}\left(\mu_{j}\right)=\sqrt{\nabla_{x}\left(\mu_{j}\right)^{2}+\nabla_{y}\left(\mu_{j}\right)^{2}+\nabla_{z}\left(\mu_{j}\right)^{2}}, \quad j \in \text { ROI }
\end{aligned}
$$

$\mathrm{TV}\left(\mu_{\mathrm{j}}\right)$ is the 1-2 norm of the image gradients. Since motion would reduce the image resolution hence the sharpness, the corresponding GV is expected to be larger for a motion-free image. As for the patient study, we only computed GV for all images as no reference was available. 
Table 1 Implementation details for each experiment.

\begin{tabular}{|c|c|c|c|c|c|c|c|}
\hline & $\begin{array}{c}\text { Simulation } \\
1 \\
\end{array}$ & $\begin{array}{c}\text { Simulation } \\
2 \\
\end{array}$ & $\begin{array}{c}\text { Simulation } \\
\mathbf{3} \\
\end{array}$ & $\begin{array}{c}\text { Simulation } \\
4 \\
\end{array}$ & $\begin{array}{c}\text { Simulation } \\
\mathbf{5} \\
\end{array}$ & Phantom & Patient \\
\hline $\begin{array}{l}\text { No. of } \\
\text { projections }\end{array}$ & 360 & 360 & 360 & 360 & 360 & 512 & 31 \\
\hline $\begin{array}{l}\text { Tube voltage } \\
\qquad(\mathrm{kVp})\end{array}$ & 1 & 1 & I & I & 1 & 90 & 90 \\
\hline $\begin{array}{l}\text { Tube current } \\
(\mathrm{mA})\end{array}$ & I & I & I & I & I & 5.0 & 5.0 \\
\hline $\begin{array}{l}\text { Image } \\
\text { matrix size } \\
\text { at full } \\
\text { resolution }\end{array}$ & $\begin{array}{c}512 \times 512 \\
\times 256\end{array}$ & $\begin{array}{c}512 \times 512 \\
\times 256\end{array}$ & $\begin{array}{c}512 \times 512 \\
\times 256\end{array}$ & $\begin{array}{c}512 \times 512 \\
\times 256\end{array}$ & $\begin{array}{c}512 \times 512 \\
\times 256\end{array}$ & $\begin{array}{c}561 \times 561 \\
\times 401\end{array}$ & $\begin{array}{c}481 \times 481 \\
\times 481\end{array}$ \\
\hline $\begin{array}{l}\text { Voxel size } \\
\qquad\left(\mathrm{mm}^{3}\right)\end{array}$ & $\begin{array}{l}0.2 \times 0.2 \\
\times 0.2\end{array}$ & $\begin{array}{l}0.2 \times 0.2 \\
\times 0.2\end{array}$ & $\begin{array}{l}0.2 \times 0.2 \\
\times 0.2\end{array}$ & $\begin{array}{c}0.2 \times 0.2 \\
\times 0.2\end{array}$ & $0.2 \times 0.2$ & $\begin{array}{c}0.25 \times 0.25 \\
\times 0.25\end{array}$ & $\begin{array}{c}0.125 \times 0.12 \times \\
0.125\end{array}$ \\
\hline $\begin{array}{c}\text { Level } 3 \\
\text { Iteration no. }\end{array}$ & 8 & 7 & 6 & 3 & & 4 & 5 \\
\hline $\begin{array}{c}\text { Level } 2 \\
\text { Iteration no. }\end{array}$ & 4 & 2 & 4 & & 2 & 2 & 2 \\
\hline $\begin{array}{c}\text { Level } 1 \\
\text { Iteration no. }\end{array}$ & 0 & 0 & 0 & 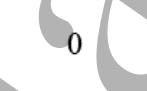 & 0 & 0 & 0 \\
\hline $\begin{array}{l}\text { Total } \\
\text { Iteration no. }\end{array}$ & 12 & 9 & 10 & & 6 & 6 & 7 \\
\hline $\begin{array}{l}\text { Computation } \\
\text { time (min) }\end{array}$ & 7.5 & 5.0 & & 4.1 & 4.8 & 36 & 25 \\
\hline
\end{tabular}

\section{Experiments}

The proposed ME/MC method was evaluated with simulations, phantom and patient studies. Parameter values used in all studies are listed in Table 1 . The details of each experiment are described below.

\subsection{Simulations}

A digital phantom (Figure 5) was used in all simulations. It was discretized into an image of $512 \times 512 \times 256$ voxéls with a voxel size of $0.2 \times 0.2 \times 0.2 \mathrm{~mm}^{3}$. A detector with $400 \times 300$ detector pixels with pixel size of $0.2 \times 0.2 \mathrm{~mm}^{2}$ was simulated to create the transaxially truncated projections. The distance between the X-ray source and the detector was $575 \mathrm{~mm}$, the distance between the detector and the rotation center was $216.5 \mathrm{~mm}$.

All simulations are noise-free with a monochromatic source energy of $70 \mathrm{keV}$. Stepand-shoot acquisition mode was simulated, with a total number of projection views 360 covering one full rotation. The projections were simulated with distance-driven projector [24], while Joseph's ray tracing projector was applied in all reconstructions. We assumed that the motion within one cone-beam projection view is negligible, hence there was no motion simulated within one projection view. Three simulated motion 
Figure 5. The digital phantom that used in the simulations. Left: selected axial view; right: coronal and sagittal views.

We compared the image quality in three cases: one reconstructed with motionfree projections as a reference, one with motion-contaminated projections but without any compensation, and one with motion-contaminated projections corrected with the proposed ME/MC method. For reference and uncorrected images, a cone-beam circular FDK algorithm was used for reconstruction. The motion corrected image was computed as follows. In one image update, high-res and low-res patches were updated in an interleaved fashion in a subiteration, as in Figure 3. The high-res and low-res patches had resolutions of $0.2 \times 0.2 \times 0.2 \mathrm{~mm}^{3}$ and $0.8 \times 0.8 \times 0.8 \mathrm{~mm}^{3}$, respectively. The total number of ME iterations depended on each scan. The multi-resolution scheme was adopted to accelerate the computation. As explained in Section 2.3, the iterative process was stopped when the relative change of the re-projection errors was below a threshold $(2 \%)$. A final corrected image was obtained with a modified FDK algorithm compensating for the estimated motion, as in Section 2.4.

We compared the proposed method with the method described in [8]. [8] applied autofocus techniques to motion estimation, which has been previously employed in CBCT for correction of géometric misalignment [26]. Motion was estimated by minimizing the motion artifact metric of the image (image entropy). The minimization was performed together with FDK reconstruction in an iterative minimization process, with respect to the number of points being used for down-sampling the trajectory. Simulation studies were performed with the motion as the ground truth was available.

\subsection{Phantom Study}

A phantom scan was performed on an 3D Accuitomo 170 scanner (Morita Inc., Kyoto, Japan) in the Oral and Maxillofacial Surgery Department of University Hospital of Leuven. The anthropomorphic phantom model was a Sectional Head Phantom SK150 
(a)

(b)

(c)

(d)

(e)
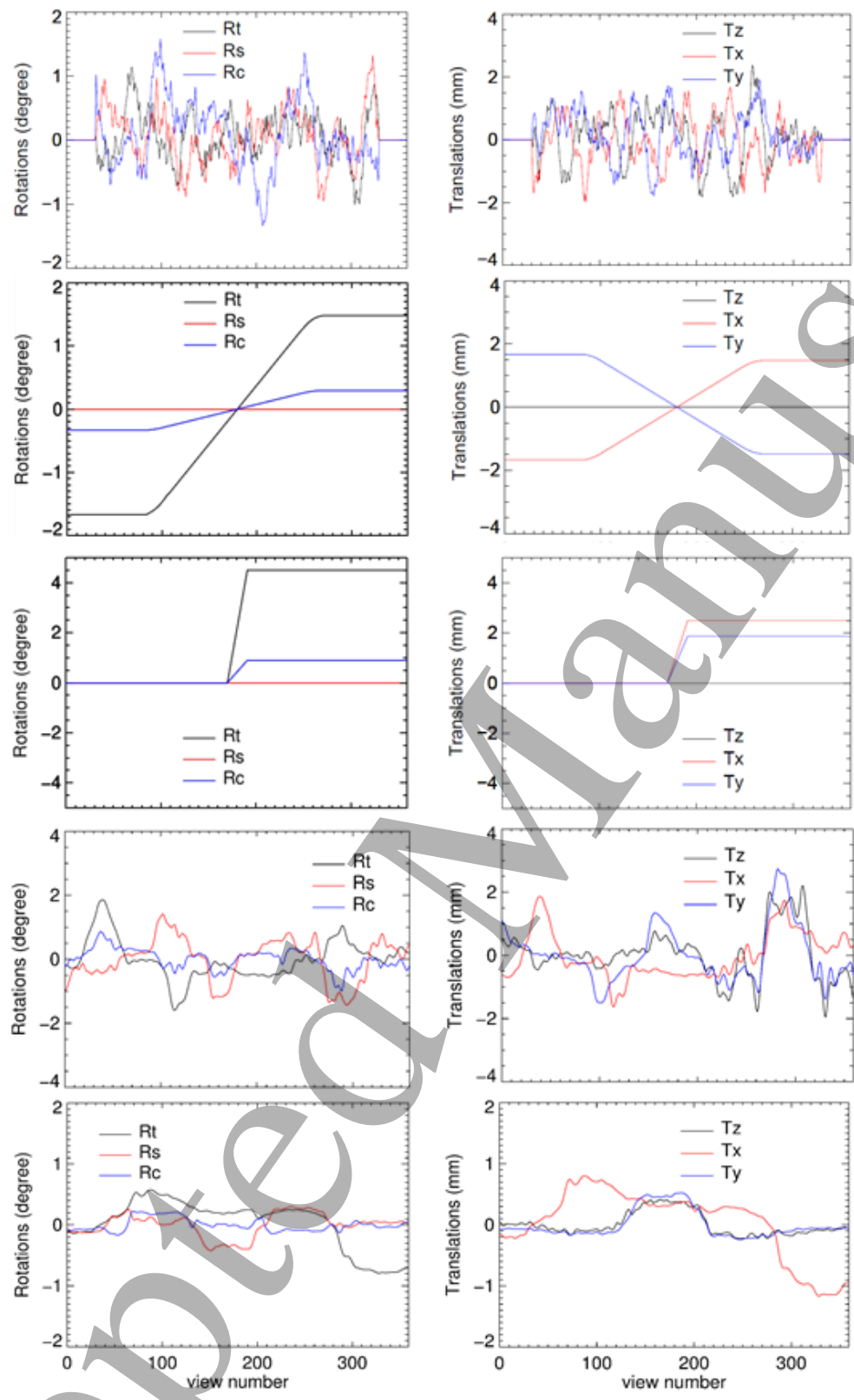

S1
S4

S5

Figure 6. The motion segments used to generate the motion-contaminated projections: (a) tremble motion S1, (b) slow-drift motion S2, (c) abrupt motion occurred in the middle of the scan S3, (d) and (e) recorded motion segments from subject S4 and S5. Rt, Rs, Rc, Tz , Tx , Ty were rotation and translation parameters defined in Figure 1. Details of how (d) and (e) were recorded can be found in [25]. 
(Phantom Laboratories, Salem, NY, USA). This phantom has an internal air cavity representing the oral, pharynx and trachea anatomy. The scan was a full rotation scan with 512 views. The total scan lasted for $17.5 \mathrm{~s}$ using continuous irradiation, with exposure time at each view was $\sim 30 \mathrm{~ms}$. Other scan parameters were: detector size $940 \times 748$, detector pixels $0.2 \times 0.2 \mathrm{~mm}^{2}$; scan FOV $140 \times 100 \mathrm{~mm}^{2}$; distance between the $\mathrm{X}$-ray source and the detector $842 \mathrm{~mm}$; distance between the detector and the rotation center $302 \mathrm{~mm}$.

Motion was induced once by pulling a string attached to the phantom in the middle of the scan, while having the phantom centered in the scanner FOV. After starting the acquisition $(\sim 5 \mathrm{~s})$, the examiner pulled the non-elastic string gently (lasted for $\sim 2 \mathrm{sec}$ ) from outside the examination room. The maximal induced amplitude was about 10 degree of rotation and $5 \mathrm{~mm}$ of translation. After the induced motion, the phantom returned to a new stable position in which it remained relatively stationary for the rest of the scan. The induced motion was expected to create motion artifacts in the reconstructed image. Following the scan with induced movement, a static scan using the same scan parameters but the movement was performed as a reference.

Similar to the simulation study, we aimed to compare the uncorrected, corrected and reference images. An image of the static scan reconstructed using the scanner software was used as the reference image. The size of this image was $561 \times 561 \times 401$ with voxel size $0.25 \times 0.25 \times 0.25 \mathrm{~mm}^{3}$. A scanner-reconstructed image of the scan with motion was exported as the uncorrected image. The dimensions of this image were the same as the reference image. No motion compensation or post-processing was applied. The motion corrected image was produced as follows. For the moving scan, raw measurement data were first exported. The high-res patch had a resolution of $0.25 \times 0.25 \times 0.25 \mathrm{~mm}^{3}$, while the low-res patch had $1 \times 1 \times 1 \mathrm{~mm}^{3}$. Multi-resolution was used to accelerate the iterative ME. MC was performed with a modified high-res FDK algorithm with estimated motion. The voxel size was the same as that of the reference image.

\subsection{Patient Study}

A 9-year-old child with cleft lip and palate underwent a CBCT scan for the planning of the follow-up treatment. The reconstructed image contained visible motion artifacts. This scan was aequired on the same scanner as the phantom study and had a full rotation acquisition with 512 views, and a scan time of $17.5 \mathrm{~s}$ with continuous irradiation, with exposure time at each view was $\sim 30 \mathrm{~ms}$. Detector size was $940 \times 748$, detector pixel was $0.2 \times 0.2 \mathrm{~mm}^{2}$, scan FOV was $100 \times 50 \mathrm{~mm}^{2}$. Other parameters were the same as the ones used in the phantom study. The image size was $481 \times 481 \times 481$, voxel size was $0.125 \times 0.125 \times 0.125 \mathrm{~mm}^{3}$. The high-res patch had a resolution of $0.125 \times 0.125 \times 0.125$ $\mathrm{mm}^{3}$, while the low-res patch had $0.5 \times 0.5 \times 0.5 \mathrm{~mm}^{3}$. Iterative $\mathrm{ME}$ was performed to estimate the motion. MC was performed in FDK with the motion accounted for during backprojection to obtain the motion-corrected image. 


\section{Results}

\subsection{Simulations}

The number of iterations and multi-resolution scheme design for each study can be seen in Table 1. Note that both motion-corrected and uncorrected images were first registered to the reference image. This was done because the estimated motion is not always identical to the true motion, resulting in positional and rotational differences with respect to the reference image. The pose of the reconstructed object is arbitrary and roughly corresponds to the average pose during the scan.

Figure 7 shows the images resulting from the simulation experiments. For all simulations with motion from Figure 6, compared to the reference image, the image without any compensation was clearly contaminated by motion artifacts. After applying $\mathrm{ME} / \mathrm{MC}$ to the measured data, most of the artifacts were suppressed. The double edges and blurring in the uncorrected images mostly disappeared. The overall shapes of the teeth were recovered and the edges were sharper after correction. The corrected images were more similar to the reference image, as indicated by the higher MSSIM (2.41\% in average), $\mathrm{GV}$ (7.71\% in average) and lower RMSE (11.64\% in average). There are some residual ghost structures in Figure $7 \mathrm{e}$, which we consider was because the motion estimation cannot fully recoyer some slow-moving component in Figure 6e. The reconstruction therefore brought structures from other axial position to the display slice position by attempting to explain the suboptimal motion estimate. Figure 8 shows the difference images obtained by subtracting the reference image from corrected and uncorrected images in selected experiments. The difference was much smaller after correction which indicates the recovery of the image quality. We performed convergence analysis for simulation with motion in Figure 6e, for which the residual projection errors at each iteration were plotted and shown in Figure 13.

The selection of the optimal LOG parameters in Eq. (5), i.e., Gaussian smoothing width $\sigma$ and Laplacian window size was determined based on the experiment in Figure 9, in which the quality index MSSIM, RMSE and GV were optimal for simulations. The final LOG parameters for motion correction were taken as the average values that resulted in optimal quality indices in all simulations. As a result, $\sigma=1.0$ and window size $=5 \times 5$ were applied across experiments. Table 2 demonstrates the superiority of the LOG filter over other first order filters. All operator parameters were optimized to achieve their best performance. All three filters enabled better image quality after correction to some extent, while LOG outperformed the other two in terms of MSSIM, RMSE and GV. Figure 14a shows the visual effects of feature-based motion estimation on recovering image quality for the simulation.

We compared the proposed method with the method described in [8]. Simulation studies were performed with the motion in Figure 6 as the ground truth was available. As in Figure 10, all three image quality indices were superior or at least comparable with our proposed method. The processing time to produce an image (with the simulated motion of Figure $6 \mathrm{~b}$ ) was $5 \mathrm{~min}$ for our method and $20.5 \mathrm{~min}$ for the method to be 
(a)

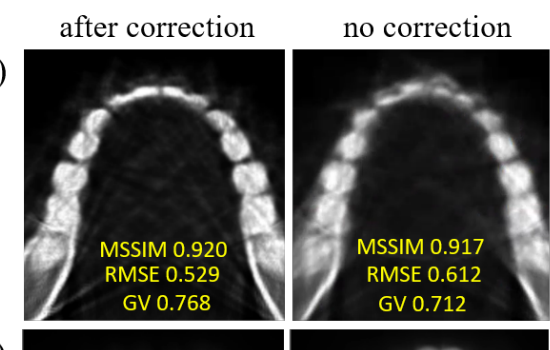

(b)

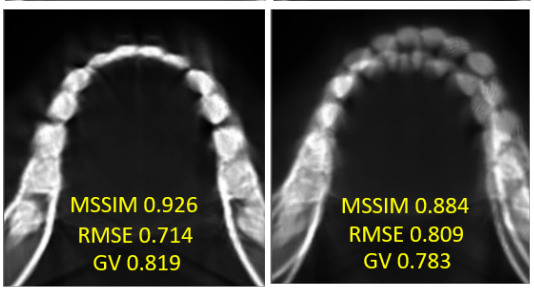

(c)

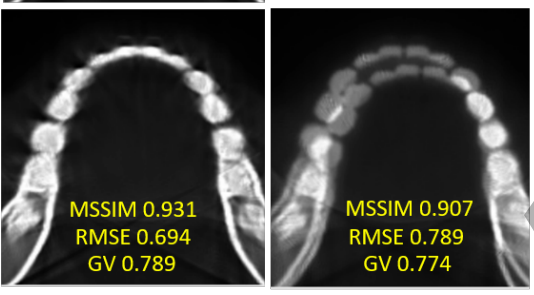

compared.

Figure 7. (a)-(e) Axial planes of simulations with motion shown in Figure 6 (each corresponds to S1-S5). (f) reference plane. After correction, most artifacts were successfully suppressed and details in the teeth were recovered. The MSSIM, RMSE $\left(\times 10^{-2}\right)$ and $\mathrm{GV}$ are listed on top of images. The yellow dashed circle in (f) contains the region where GV was calculated. The red dashed circle in (g) contains the FOV. The display window is $\left[-0.01,0.05 \mathrm{~cm}^{-1}\right]$. 

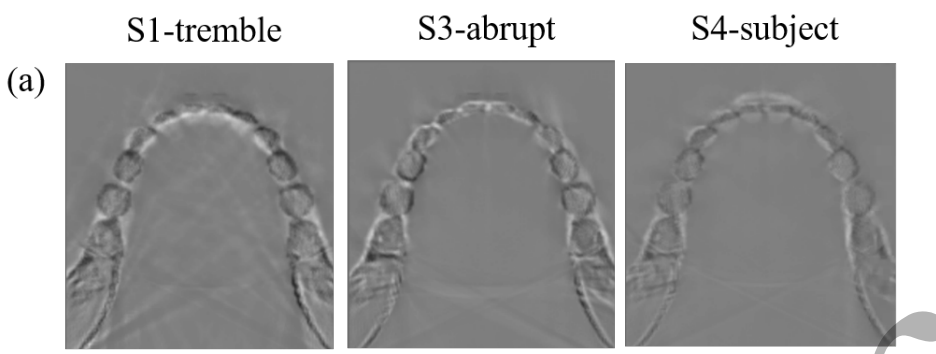

(b)
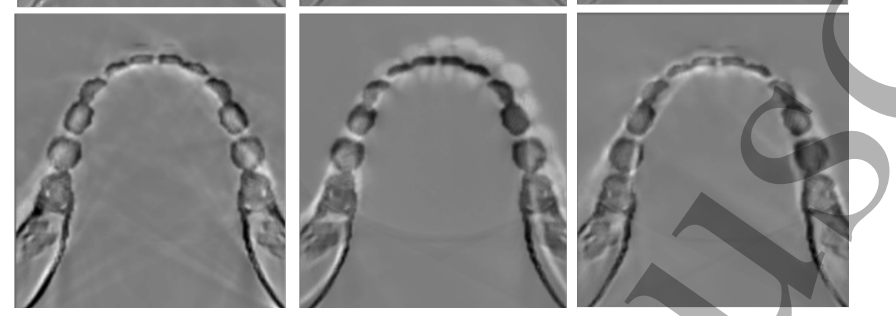

Figure 8. Axial planes of differences between (a) the corrected, (b) the uncorrected image and the reference images. Note here we only show results in three experiments, with the presence of tremble (S1), abrupt (S3) and real subject (S4) motion in Figure 6. After motion correction, the image quality was recovered significantly as indicated by the reduced difference from the reference image. The display window is $[-0.05,0.05$ $\left.\mathrm{cm}^{-1}\right]$.

Table 2 Comparison of applying different filtering on projections for simulations with motions in Figure 6. "None" indicates no filtering was applied. The number in bold indicates the index with best performance. LOG operations on projections enable recovering the best image quality.

\begin{tabular}{|c|c|c|c|c|c|}
\hline & Motion & LOG & Sobel & Robert & None \\
\hline \multirow{10}{*}{ MSSIM } & & 0.920 & 0.923 & 0.913 & 0.919 \\
\hline & & 0.926 & 0.919 & 0.912 & 0.918 \\
\hline & & 0.931 & 0.928 & 0.930 & 0.926 \\
\hline & & 0.935 & 0.919 & 0.934 & 0.931 \\
\hline & S5 & 0.924 & 0.915 & 0.922 & 0.920 \\
\hline & S1 & 0.529 & 0.544 & 0.551 & 0.549 \\
\hline & S2 & 0.714 & 0.734 & 0.740 & 0.746 \\
\hline & S3 & 0.694 & 0.729 & 0.802 & 0.732 \\
\hline & $\mathrm{S} 4$ & 0.568 & 0.566 & 0.612 & 0.577 \\
\hline & S5 & 0.551 & 0.565 & 0.575 & 0.568 \\
\hline \multirow{5}{*}{$\mathrm{GV}$} & S1 & 0.768 & 0.765 & 0.725 & 0.756 \\
\hline & S2 & 0.819 & 0.812 & 0.821 & 0.814 \\
\hline & S3 & 0.789 & 0.787 & 0.765 & 0.776 \\
\hline & $\mathrm{S} 4$ & 0.822 & 0.825 & 0.801 & 0.807 \\
\hline & S5 & 0.874 & 0.832 & 0.827 & 0.835 \\
\hline
\end{tabular}




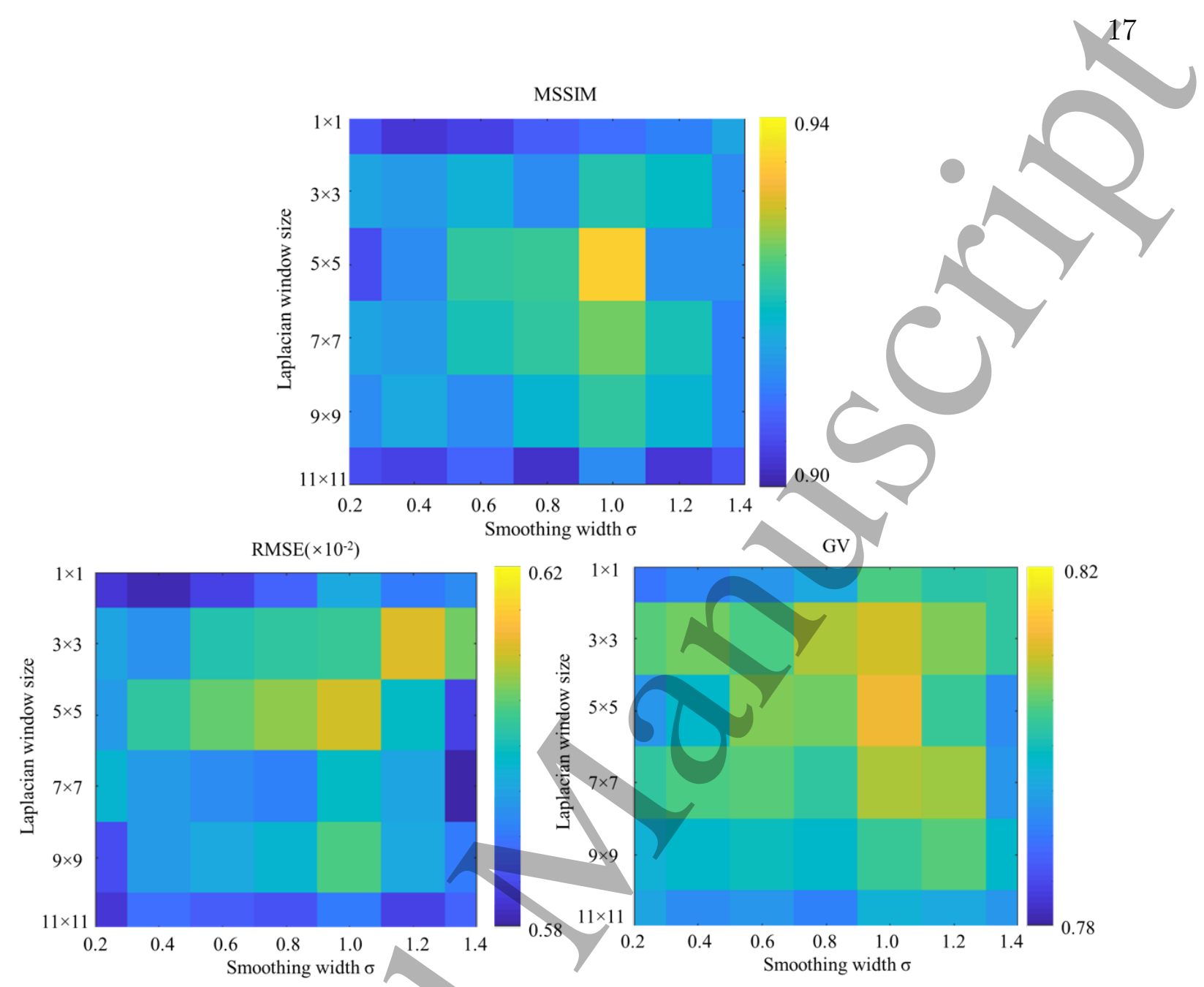

Figure 9. The optimal parameters for LOG filtering (Gaussian smoothing width $\sigma$ and Laplacian kernel window size) were found by searching the ones which resulting the largest MSSIM, together with smallest RMSE and largest GV for all simulations. The value (block) for each possible parameter setup is the average over all simulations with motions in Figure 6.

\subsection{Phantom Study}

Similar to the simulation studies, both motion-corrected and uncorrected images were first registered to the reference image. ME updated the image and the motion alternately, and a total of six iterations were performed (Table 1). Figure 11 shows the motion estimate and selected planes of these images. Compared to the reference image, the image without any compensation was clearly contaminated by motion artifacts. After applying the proposed compensation method, most of the artifacts were suppressed. Quantitative analysis indicated that the correction indeed recovered the image quality. Figure 11 contains profile plots across a tooth that show the local differences. To quantify the change of the sharpness, we also computed the GV in 
(a)

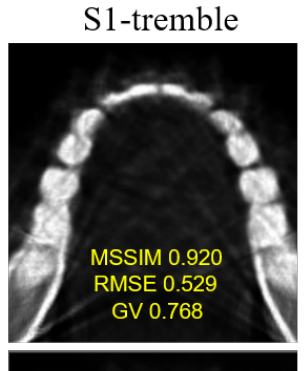

(b)
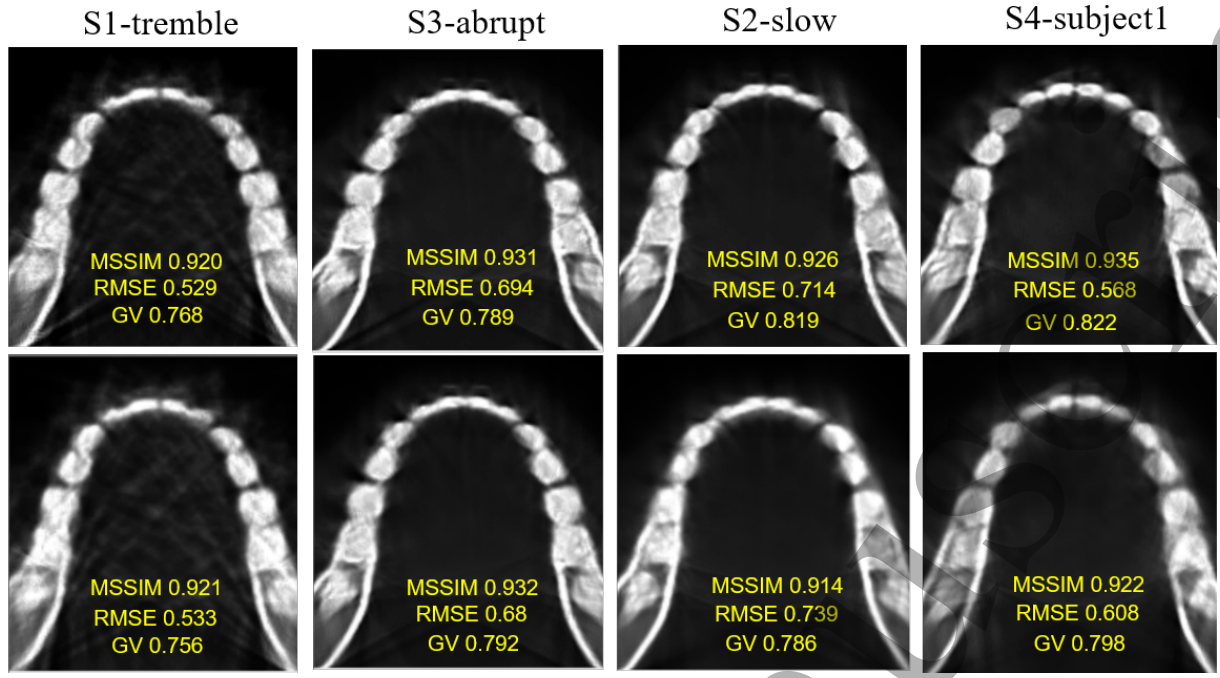

Figure 10. Comparison of MSSIM, RMSE $\left(\times 10^{-2}\right)$ and GV between the corrected images produced from (a) proposed method and (b) the ones from a compared method described in Section 2.6. Two methods produced comparable results in terms of the recovered image quality when the motion pattern was relatively abrupt and random, and the proposed method has better results when motion pattern was slow or complex.

three selected ROIs (containing one right molar, left molar and incisor, respectively, see Figure 11) for all images. GV of the teeth was increased after motion compensation (Table 3). The convergence analysis that demonstrates the residual projection error at each iteration is shown in/Figure 13. Figure 14b shows the effects of feature-based motion estimation on recovering image quality for the phantom study.

\subsection{Patient Study}

Because there was no reference (repeat) scan performed in the patient study, we only compared the motion-corrected image with the scanner reconstructed image. ME updated the image and the motion alternately, and a total of seven iterations were performed (Table 1). Figure 12 shows selected axial and coronal planes of the corrected and uncorrected images. Two dentists assessed the reconstructed images. After applying the proposed method, most of the artifacts were suppressed, and certain diagnostic features were more visible, as indicated by the red arrows in Figure 12. Dentists could identify the contact between the front teeth in the axial views after motion compensation but not before; also, structures of teeth could be better differentiated in coronal views which is useful in diagnosis of dental pathology. The image sharpness was also measured in a similar way to the phantom study. In three selected ROIs (containing one right molar, left molar, incisor), GV increased considerably (at least 18.4\%) after compensation, which indicates an increase of image sharpness (Table 3). We performed convergence analysis, which assessed the residual projection error at each iteration as shown in Figure 13. Figure 14c shows the effects of feature-based motion estimation on recovering image quality for the patient study. 
Table 3 Gradient variance $\left(\times 10^{8}\right)$ as index of image sharpness in teeth.

\begin{tabular}{ccccc}
\hline & reference & $\begin{array}{c}\text { no } \\
\text { correction }\end{array}$ & $\begin{array}{c}\text { after } \\
\text { correction } \\
\text { (no LOG) }\end{array}$ & $\begin{array}{c}\text { after } \\
\text { correction } \\
(\mathbf{w} / \mathbf{L O G})\end{array}$ \\
\hline $\begin{array}{c}\text { right molar } \\
\text { (phantom) }\end{array}$ & 1.723 & 1.019 & 1.398 & 1.515 \\
$\begin{array}{c}\text { left molar } \\
\text { (phantom) }\end{array}$ & 0.830 & 0.103 & 0.478 & 0.562 \\
$\begin{array}{c}\text { incisor } \\
\text { (phantom) }\end{array}$ & 1.203 & 0.607 & 0.901 & 0.921 \\
$\begin{array}{c}\text { right molar } \\
\text { (patient) }\end{array}$ & $/$ & 0.391 & 0.436 & 0.463 \\
$\begin{array}{c}\text { left molar } \\
\text { (patient) }\end{array}$ & $/$ & 0.601 & 1.098 & 1.188 \\
$\begin{array}{c}\text { incisor } \\
\text { (patient) }\end{array}$ & $/$ & 0.221 & 0.339 & 0.425 \\
\hline
\end{tabular}

(b)
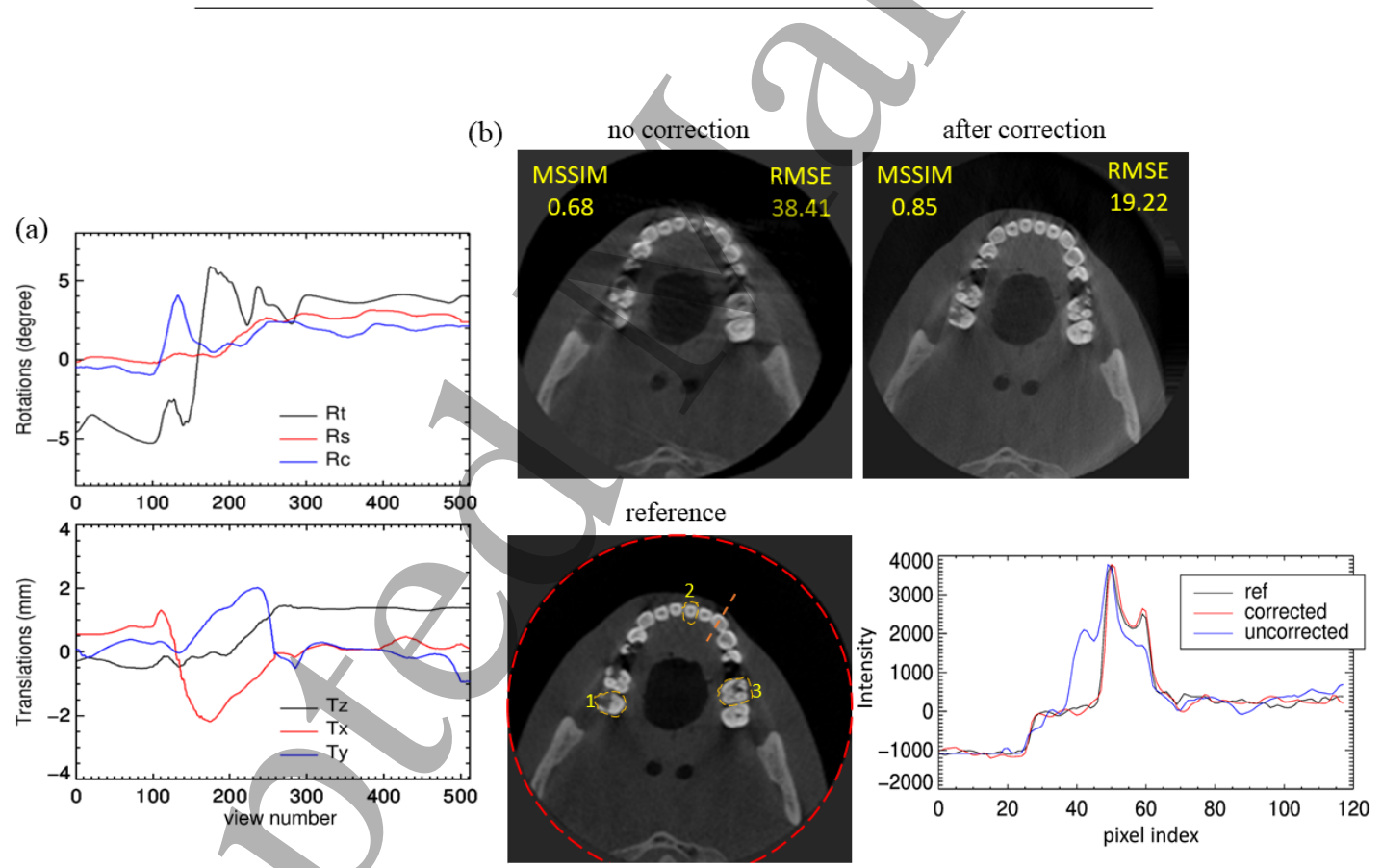

Figure 11. (a) Motion estimate from the phantom study: up are rotations, bottom are translations. (b) 2D axial planes of the reference, uncorrected and corrected images. Note that the "double edge" motion artifacts in the uncorrected image disappeared in the corrected one. MSSIM and RMSE were only calculated within a circular region containing all teeth. Both dentists agreed that better visualization of bony structures was achieved after compensation. The three teeth measured for sharpness are labeled with number. Note that the background grey circle region is outside the FOV (red dashed circle), hence is not of clinical interest. The display window is [-100, $3000 \mathrm{HU}]$. 

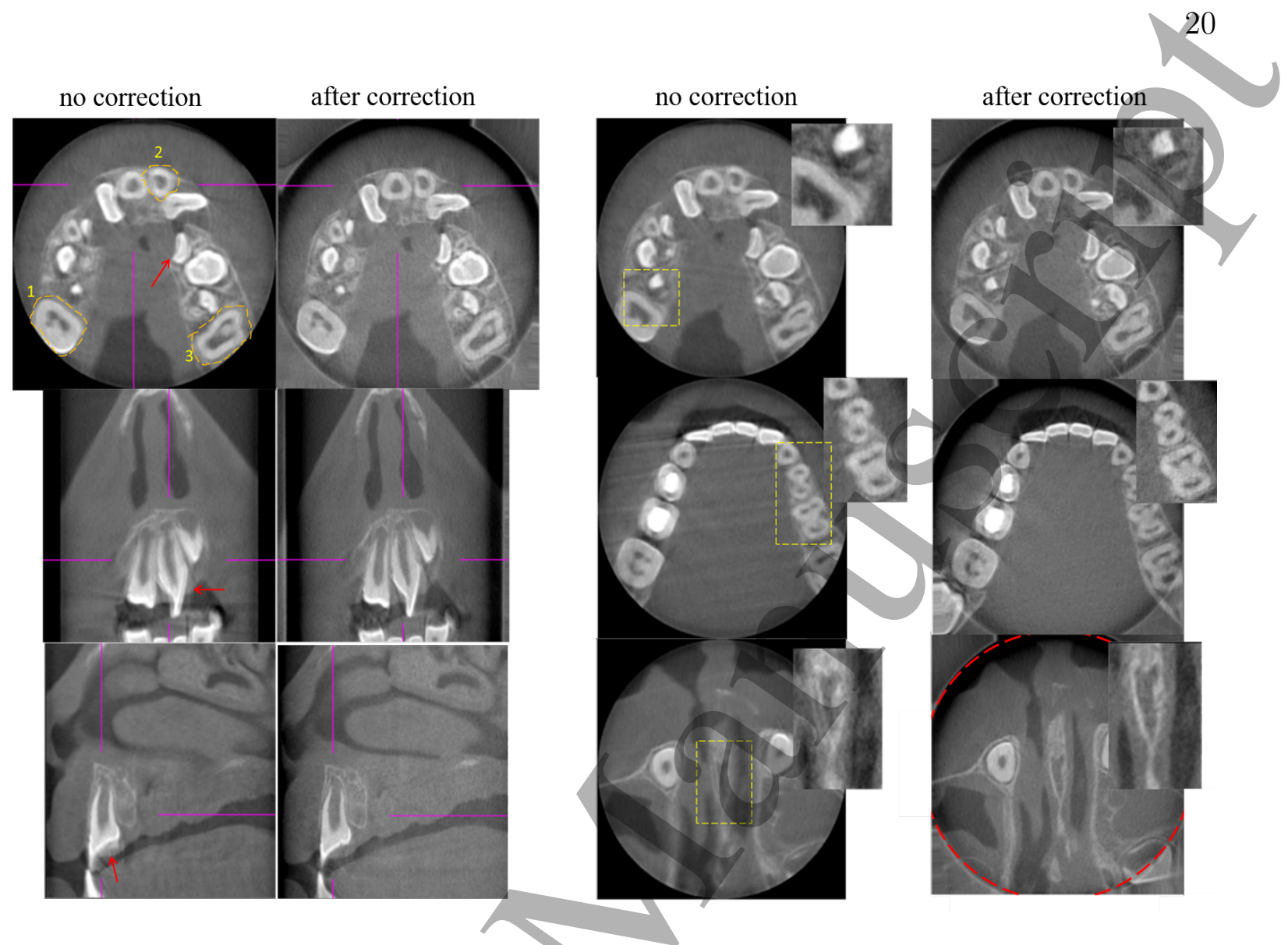

Figure 12. After applying compensation, blurring and shading artifacts were reduced substantially. As for diagnostic difference, dentists could identify the contact between the front teeth (arrow) in the axial views after motion compensation but not before; also, structures of teeth (arrow) could be better differentiated in coronal views which is useful in diagnosis of dental pathology. Three teeth measured with sharpness are labeled with number by their side. The red dashed circle indicates the FOV. The display window is $[-600,2800 \mathrm{HU}]$.

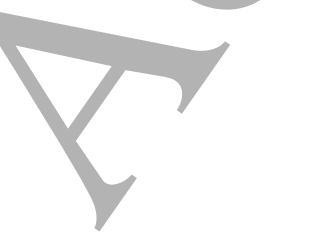

Figure 13. Residual projection errors at each iteration, obtained by summing the absolute differences between the measured and calculated projections for all pixels. Because of the different pattern and degree of the motion in each experiment, different number of iterations may be required for ME. Most of the error was eliminated after the first few iterations, where the resolution level is low. As the iteration/ resolution levels increase, the residual error tends to stabilize. 


\section{DISCUSSION}

Motion has been shown to have big impact on the high-resolution CBCT image quality $[4,5,27]$. In this paper, we proposed a motion estimation and motion compensation approach for oral and maxillofacial CBCT imaging. It tolerates transaxial truncation and only requires the measured raw data. Since no additional measurements are needed, it can be applied retrospectively to motion-contaminated scan data. The proposed method was evaluated in simulation, phantom and patient studies. After compensation, the quality of the reconstructed image was significantly improved. The method would avoid the need for repeat scans when the patient moves, which could significantly reduce the radiation exposure, especially for children who are more likely to move than adults. This is even more important for a child with cleft clip and palate who often needs a series of scans at their early ages to guide the treatments.

Note that in all cases, the motion was assumed and modeled as rigid motion of an object across all projection views. In practice, non-rigid movement of the tissues due to swallowing, or different motion of the upper and lower jaws, might be present 
in a scan. How to deal with such movements is beyond the scope of this paper. Also, together with motion, the presence of dental implants and other metallic objects like fillings and crowns could complicate the artifacts in a reconstructed image. A metal artifact correction technique $[28,29]$ could have to be combined with the proposed motion correction technique to mitigate the motion and metal artifacts. Perhaps the biggest issue is how to separate the compound effects on image quality from motion and metal. For example in [28], a method was proposed in which the motion was first estimated using an image with preliminary metal artifact reduction. Then full metal artifact reduction was performed by modeling the energy model in a polychromatic reconstruction.

The proposed approach worked reasonably well despite severe artifacts outside the high-res patch, where the reconstruction is handicapped by a limited angle problem. It has been previously shown that the high-res patch can in principle be reconstructed exactly if it contains a portion of air background (intensity known to be zero) [30], which is often the case in dental imaging. Moreover, even in the background, spatial frequencies are better reconstructed if they contribute more information to the measured projections (typically edges which are parallel to the projection lines). This implies that the reconstructed low-res patch still contains the relevant edges needed to align measured and forward projected views, which is the essence of the $\mathrm{ME} / \mathrm{MC}$ scheme.

Unlike a clinical helical CT scan, a typical oral and maxillofacial scan has a small number of views $(300 \sim 700)$ acquired in a full or half rotation. Hence the computation requirement to apply motion correction is less demanding. The entire $\mathrm{ME} / \mathrm{MC}$ process took 25 mins (20 mins for ME iterations and 5 mins for the MC/ final reconstruction) for the patient scan. Note that only forward and backward projection processes ran on GPU (NVIDIA k40), so the processing time can still be further reduced. Regarding the time reduction of patch reconstruction and multi-resolution, we found that patch reconstruction alone reduced the total computation time to $1 / 4$, while multi-resolution ME reduce the time to $1 / 10$ in a simulation study. The combination of the two accelerations achieved a 40-fold time reduction. Similar results are expected in phantom and patient studies.

There were still some remaining streak artifacts and blurring in the corrected images. These are possibly due to several limitations of the proposed method: 1) scattering and beam hardening effects were not corrected; 2) during the ME iterations, the entire object is not reconstructed exactly due to the truncation, which may reduce the estimation accuracy; 3) we assumed the movement only happens in between views. Because the motion during the acquisition of a single view is not compensated for, fast motions may produce a residual loss of resolution in the corrected images. Given the fact that the lag time in between views was negligible and the exposure time of one projection view in CBCT is $\sim 30 \mathrm{~ms}$, such movement within views might not be negligible. 4) the final FDK reconstruction is not exact when motion compensation is incorporated. However, we considered the overall motion in an oral and maxillofacial scan to be small since physical restraining during the scan already reduce the possibility 
1

2

3

4

5

6

7

8

9

of large movements. Therefore, one can use an analytical algorithm (e.g. FDK), with a first-order motion compensation during the back-projection step, without introducing significant artifacts. When motion is large, a dedicated FDK [10,31, 32] or an iterative reconstruction algorithm may be more appropriate for the final motion-compensated reconstruction and intermediate images in ME.

Variations in scan protocol could affect the motion correction. For example, a small FOV captures less information in the measurement and increases the extent of truncation, which may degrade the results. Offset scan mode is available on some scanners. With this scan mode, a small detector is used to scan a relatively large FOV, at the cost of increased and asymmetrical truncation in each view. These asymmetrically truncated projections may present a challenge to motion estimation. It is expected that a half scan typically suffers more from cone-beam artifacts, but less from motion artifacts due to the shorter scan time, compared with a full rotation scan. Meanwhile, if FDK reconstruction is used, the more consistent data of a short scan will provide reduced susceptibility to motion artifacts than a full scan, in which inconsistencies could strongly impact the image quality.

\section{CONCLUSION}

In this study, we proposed an approach to suppress the motion artifacts in oral and maxillofacial CBCT imaging. Results from simulations, phantom and patient studies were presented. The improvement in image quality was assessed both quantitatively and qualitatively. Further clinical evaluation with different scan protocol setups is needed. 


\section{ACKNOWLEDGEMENTS}

The authors want to thank Dr. Koen Michielsen and Dr. Guozhi Zhang for providing the digital phantom and helpful discussion.

\section{References}

[1] E. Y. Keriş, Effect of patient anxiety on image motion artefacts in CBCT, BMC Oral Health 17 (2017) 1-9. doi:10.1186/s12903-017-0367-4. URL https://dx.doi.org/10.1186/s12903-017-0367-4

[2] R. Spin-Neto, L. H. Matzen, L. Schropp, E. Gotfredsen, A. Wenzel, Factors affecting patient movement and re-exposure in cone beam computed tomography examination, Oral Surgery, Oral Medicine, Oral Pathology and Oral Radiology 119 (5) (2015) 572-578. doi:https://doi.org/10.1016/j.oooo.2015.01.011.

[3] K. Donaldson, S. O'Connor, N. Heath, Dental cone beam CT image quality possibly reduced by patient movement, Dentomaxillofacial Radiology 42 (2) (2013) 91866873-91866873. doi: $10.1259 / \mathrm{dmfr} / 91866873$.

URL https://dx.doi.org/10.1259/dmfr/91866873

[4] R. Spin-Neto, A. Wenzel, Patient movement and motion artefacts in cone beam computed tomography of the dentomaxillofacial region: a systematic literature review, Oral Surgery, Oral Medicine, Oral Pathology and Oral Radiology 121 (2016) 425-433. doi:10.1016/j.oooø.2015.11.019.

URL https://dx.doi.org/10.1016/j.oooo.2015.11.019

[5] R. Pauwels, K. Araki, J. H. Siewerdsen, S. S. Thongvigitmanee, Technical aspects of dental CBCT: state of the art, Dentomaxillofacial Radiology 44 (1) (2015) 20140224-20140224. doi:10.1259/dmfr.20140224. URL https://dx.doi.org/10.1259/dmfr.20140224

[6] C. Nardi, C. Borri, F. Regini, L. Calistri, A. Castellani, C. Lorini, S. Colagrande, Metal and motion artifacts by cone beam computed tomography (CBCT) in dental and maxillofacial study, La radiologia medica 120 (7) (2015) 618-626. doi:10.1007/s11547-015-0496-2. URL https://dx.doi.org/10.1007/s11547-015-0496-2

[7] T. Hanzelka, J. Dusek, F. Ocasek, J. Kucera, J. Sedy, J. Benes, G. Pavlikova, R. Foltan, Movement of the patient and the cone beam computed tomography scanner: objectives and possible solutions, Oral Surgery, Oral Medicine, Oral Pathology and Oral Radiology 116 (2013) 769-773. doi:10.1016/j.oooo.2013.08.010.

[8] J. Wicklein, Y. Kyriakou, W. A. Kalender, H. Kunze, An online motion-and misalignment-correction method for medical flat-detector CT, 86681S). International Society for Optics and Photonics 8668 (2013).

[9] A. Sisniega, J. W. Stayman, J. Yorkston, J. H. Siewerdsen, W. Zbijewski, Motion compensation in extremity cone-beam CT using a penalized image sharpness criterion, Physics in Medicine and Biology 62 (9) (2017) 3712-3734. doi:10.1088/1361-6560/aa6869.

URL https://dx.doi.org/10.1088/1361-6560/aa6869

[10] S. Jang, S. Kim, M. Kim, J. B. Ra, Head motion correction based on filtered backprojection for x-ray CT imaging, Medical Physics 45 (2) (2018) 589-604. doi:10.1002/mp.12705. URL https://dx.doi.org/10.1002/mp.12705

[11] H. Bruder, C. Rohkohl, K. Stierstorfer, T. Flohr, Compensation of skull motion and breathing motion in CT using data-based and/image-based metrics, respectively, SPIE Medical Imaging 9783 (2016) 97831-97831.

[12] S. Ouadah, M. Jacobson, J. W. Stayman, T. Ehtiati, C. Weiss, J. H. Siewerdsen, Correction of patient motion in cone-beam CT using 3D-2D registration, Phys Med Biol 62 (23) (2009) 8813-8831.

[13] Y. Ko, S. Moon, J. Baek, H. Shim, Rigid and non-rigid motion artifact reduction in X-ray CT using attention module, Medical Image Analysis 67 202-202.

[14] R. Spin-Neto, L. H. Matzen, L. Schropp, E. Gotfredsen, A. Wenzel, Detection of patient movement during CBCT examination using video observation compared with an accelerometer-gyroscope tracking

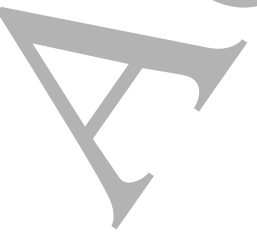

April 17, 2021 
system, Dentomaxillofacial Radiology 46 (2) (2017) 20160289-20160289. doi:10.1259/dmfr.20160289. URL https://dx.doi.org/10.1259/dmfr.20160289

[15] M. E. Eldib, M. A. Hegazy, M. H. Cho, M. H. Cho, S. Y. Lee, A motion artifact reduction method for dental CT based on subpixel-resolution image registration of projection data, Computers in Biology and Medicine 103 (2018) 232-243. doi:10.1016/j.compbiomed.2018.10.028. URL https://dx.doi.org/10.1016/j.compbiomed.2018.10.028

[16] T. Sun, J.-H. Kim, R. Fulton, J. Nuyts, An iterative projection-based motion estimation and compensation scheme for head x-ray CT, Medical Physics 43 (10) (2016) 5705-5716. doi:10.1118/1.4963218. URL https://dx.doi.org/10.1118/1.4963218

[17] K. V. Slambrouck, J. Nuyts, Reconstruction Scheme for Accelerated Maximum Likelihood Reconstruction: The Patchwork Structure, IEEE Transactions on Nuclear Science 61 (1) (2014) 173-181. doi:10.1109/tns.2013.2287637. URL https://dx.doi.org/10.1109/tns.2013.2287637

[18] K. V. Slambrouck, J. Nuyts, Metal artifact reduction in computed tomography using local models in an image block-iterative scheme, Medical Physics 39 (11) (2012) 7080-7093. doi:10.1118/1.4762567. URL https://dx.doi.org/10.1118/1.4762567

[19] J. A. Fessler, E. P. Ficaro, N. H. Clinthorne, K. Lange, Grouped-coordinate ascent algorithms for penalized-likelihood transmission image reconstruction, IEEE Transactions on Medical Imaging 16 (2) (1997) 166-175. doi:10.1109/42.563662.

URL https://dx.doi.org/10.1109/42.563662

[20] L. Yu, Y. Zou, E. Y. Sidky, C. A. Pelizzari, P. Munro, X. Pan, Region of interest reconstruction from truncated data in circular cone-beam CT, IEEE Transactions on Medical Imaging 25 (7) (2006) 869881. doi:10.1109/tmi.2006.872329. URL https://dx.doi.org/10.1109/tmi.2006.872329

[21] L. A. Feldkamp, L. C. Davis, J. W. Kress, Practical cone-beam algorithm, Journal of the Optical Society of America A 1 (6) (1984) 612-612. doi:10.1364/josaa.1.000612. URL https://dx.doi.org/10.1364/josaa.1.000612

[22] Z. Wang, A. C. Bovik, H. R. Sheikh, E. P. Simoncelli, Image Quality Assessment: From Error Visibility to Structural Similarity, IEEE Transactions on Image Processing 13 (4) (2004) 600-612. doi:10.1109/tip.2003.819861. URL https://dx.doi.org/10.1109/tip.2003.819861

[23] M. A. Bueno-Ibarra, J. Alvarez-Borrego, L. Acho, C.-S. Mc, Fast autofocus algorithm for automated microscopes, Opt Eng 44 (6) (2005) 63601-63601.

[24] B. D. Man, S. Basu, Distance-driven projection and backprojection in three dimensions, Physics in Medicine and Biology 49 (11) (2004) 2463-2475. doi:10.1088/0031-9155/49/11/024. URL https://dx.doi.org/10.1088/0031-9155/49/11/024

[25] J. H. Kim, T. Sun, A. R. Alcheikh, Z. Kuncic, J. Nuyts, R. Fulton, Correction for human head motion in helical x-ray CT, Physics in Medicine and Biology 61 (4) (2016) 1416-1438. doi:10.1088/0031$9155 / 61 / 4 / 1416$. URL https://dx.doi.org/10.1088/0031-9155/61/4/1416

[26] Y. Kyriakou, R. M. Lapp, L. Hillebrand, D. Ertel, W. A. Kalender, Simultaneous misalignment correction for approximate circular cone-beam computed tomography, Physics in Medicine and Biology 53 (22) (2008) 6267-6289. doi:10.1088/0031-9155/53/22/001. URL https://dx.doi.org/10.1088/0031-9155/53/22/001

[27] L. Li, Z. Chen, X. Jin, H. Yu, G. Wang, Experimental measurement of human head motion for highresolution computed tomography system design, Optical Engineering 49 (6) (2010) 63201-63201.

[28] T. Sun, J. Nuyts, R. Fulton, Simultaneous correction of motion and metal artifacts in head CT scanning, IEEE Nuclear Science Symposium and Medical Imaging Conference 2017 1-3.

[29] A. Hahn, M. Knaup, M. Brehm, S. Sauppe, M. Kachelrieß, Two methods for reducing moving metal artifacts in cone-beam CT, Medical Physics 45 (8) (2018) 3671-3680. doi:10.1002/mp.13060. URL https://dx.doi.org/10.1002/mp.13060

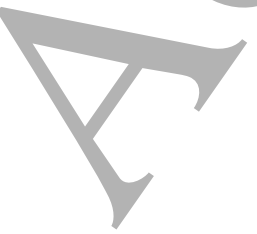

April 17, 2021 
[30] M. Defrise, F. Noo, R. Clackdoyle, H. Kudo, Truncated Hilbert transform and image reconstruction from limited tomographic data, Inverse Problems 22 (3) (2006) 1037-1053. doi:10.1088/0266$5611 / 22 / 3 / 019$.

URL https://dx.doi.org/10.1088/0266-5611/22/3/019

[31] T. Sun, R. Fulton, J. Nuyts, A method to reduce the data-redundancy artifacts for arbitrary source trajectories in CT imaging, IEEE Nuclear Science Symposium and Medical Imaging Conference 2018 (2018) $1-3$.

[32] J. Nuyts, R. Fulton, Iterative FDK reconstruction for helical CT of the head with rigid motion compensation, The 6th International Conference on Image Formation in X-Ray Computed Tomography (2020) 248-251. 